\title{
Perfil epidemiológico do trauma buco-maxilo-facial em vítimas de agressão física
}

\author{
Epidemiological profile of oral-maxillofacial in victims of physical aggression \\ Perfil epidemiológico del trauma oral-maxilofacial em víctimas de agresión física
}

Carlos Eduardo dos Santos

ORCID: https://orcid.org/0000-0002-8205-5114 Universidade Federal de Alagoas, Brasil

E-mail: santoscarlossantos@outlook.com

Diogo Gomes Brandão

ORCID: https://orcid.org/0000-0001-6167-0399

Universidade Federal de Alagoas, Brasil

E-mail: diogo.brandao2009@ hotmail.com

Silvia Girlane Nunes da Silva

ORCID: https://orcid.org/0000-0002-9319-3811

Universidade Federal de Alagoas, Brasil

E-mail: silviagirlane@hotmail.com

Ana Caroliny do Nascimento Oliveira

ORCID: https://orcid.org/0000-0001-8660-2245 Universidade Federal de Alagoas, Brasil E-mail: olivices@gmail.com

Gabriela de Almeida Sousa

ORCID: https://orcid.org/0000-0001-8734-4831 Universidade Federal de Alagoas, Brasil

E-mail: ggabrielalmeida1@gmail.com

Tallisson Emmanuel Silva de Lucena

ORCID: https://orcid.org/0000-0003-2871-0728 Universidade Federal de Alagoas, Brasil E-mail: tallisson-lucena@ hotmail.com

Iris Marilia Alves da Silva

ORCID: https://orcid.org/0000-0001-5396-213X Universidade Federal de Alagoas, Brasil

E-mail: irismariliaas@gmail.com

Érika Priscila Santos Melo

ORCID: https://orcid.org/0000-0003-1102-2208 Universidade Federal de Alagoas, Brasil

E-mail: erikamelomed@gmail.com

Kelly Rodrigues Mota

ORCID: https://orcid.org/0000-0002-1656-608X Universidade Federal de Alagoas, Brasil

E-mail: kellyrmota@outlook.com

\begin{abstract}
Resumo
Devida a sua posição de vulnerabilidade no corpo, a face, é uma região anatômica com foco de alta incidência em agressões físicas e acidentes. Segundo a Organização Mundial de Saúde (OMS) o trauma está entre as principais causas de morte e invalidez do mundo, aliado ao alarmante o crescimento de todas as formas de violência em nossa sociedade atual, principalmente a agressão física interpessoal. Embora esta modalidade de crime na sua maioria não resulte em morte, são responsáveis por significativas sequelas. Este trabalho trata-se de uma revisão integrativa, com objetivo de analisar estudos sobre o perfil epidemiológico do trauma buco-maxilo-facial em vítimas de agressão física. Os artigos compilados neste estudo foram selecionados por meio das bases de dados: PubMed, Lilacs e Scielo. O levantamento compreendeu todas as publicações encontradas, segundo as bases de dados supracitadas, sem recorte temporal até o ano de 2020. Por fim, nenhuma restrição de idioma foi aplicada, utilizando os descritores padronizados: Agressão; Lesões Maxilofaciais; Epidemiologia e seus correspondentes em inglês. Após análise dos trabalhos, observou-se que as vítimas de agressão física, indicaram uma predominância do sexo masculino, a incidência das agressões prevaleceu entre a segunda e terceira década de vida, no período noturno, sobre as fraturas ósseas recorrentes em agressões físicas, o destaque é para o complexo anatômico nasal, região mandibular e do complexo zigomático-maxilar, marcadamente no terço médio da face. Por fim, fica evidente a necessidade de uma profícua atenção a estes dados que podem beneficiar a sociedade ao fundamentar estratégias governamentais preventivas para controlar os eventos que podem resultar em traumatismos desta etiologia.
\end{abstract}

Palavras-chave: Agressão; Lesões maxilofaciais; Epidemiologia. 


\begin{abstract}
Due to its position of vulnerability in the body, the face is an anatomical region with a high incidence of physical aggression and accidents. According to the World Health Organization (WHO), trauma is among the main causes of death and disability in the world, together with the alarming growth of all forms of violence in our society today, especially interpersonal physical aggression. Although this type of crime for the most part does not result in death, they are responsible for significant sequelae. This work is an integrative review, aiming to analyze studies on the epidemiological profile of oral and maxillofacial trauma in victims of physical aggression. The articles compiled in this study were selected using the following databases: PubMed, Lilacs and Scielo. The survey included all the publications found, according to the aforementioned databases, without a time frame until 2020. Finally, no language restriction was applied, using the standardized descriptors: Aggression; Maxillofacial Lesions; Epidemiology and its English counterparts. After analyzing the works, it was observed that victims of physical aggression indicated a predominance of males, the incidence of aggression prevailed between the second and third decade of life, at night, over recurrent bone fractures in physical aggression, the highlight is for the nasal anatomical complex, mandibular region and the zygomatic-maxillary complex, markedly in the middle third of the face. Finally, it is evident the need for a fruitful attention to these data that can benefit society by supporting preventive government strategies to control the events that can result in trauma of this etiology.
\end{abstract}

Keywords: Aggression; Maxillofacial lesions; Epidemiology.

\title{
Resumen
}

Por su posición de vulnerabilidad en el cuerpo, el rostro es una región anatómica con una alta incidencia de agresiones físicas y accidentes. Según la Organización Mundial de la Salud (OMS), el trauma se encuentra entre las principales causas de muerte y discapacidad en el mundo, junto con el alarmante crecimiento de todas las formas de violencia en nuestra sociedad actual, especialmente la agresión física interpersonal. Aunque este tipo de delitos en su mayoría no resultan en muerte, son responsables de importantes secuelas. Este trabajo es una revisión integradora, con el objetivo de analizar estudios sobre el perfil epidemiológico del trauma oral-maxilofacial en víctimas de agresión física. Los artículos recopilados en este estudio fueron seleccionados utilizando las siguientes bases de datos: PubMed, Lilacs y Scielo. La encuesta incluyó todas las publicaciones encontradas, según las bases de datos antes mencionadas, sin un marco temporal hasta 2020. Finalmente, no se aplicó restricción de idioma, utilizando los descriptores estandarizados: Agresión; Lesiones maxilofaciales; Epidemiología y sus contrapartes inglesas. Luego de analizar los trabajos, se observó que las víctimas de agresión física indicaron un predominio del sexo masculino, la incidencia de agresión predominó entre la segunda y tercera década de la vida, en la noche, sobre las fracturas óseas recurrentes en la agresión física, el destaque es para la nasal. complejo anatómico, región mandibular y complejo cigomático-maxilar, marcadamente en el tercio medio de la cara. Finalmente, es evidente la necesidad de una atención fructífera a estos datos que pueden beneficiar a la sociedad al apoyar estrategias gubernamentales preventivas para controlar los eventos que pueden resultar en traumas de esta etiologia.

Palabras clave: Agresión; Lesiones maxilofaciales; Epidemiología.

\section{Introdução}

É alarmante o crescimento de todas as formas de violência em nossa sociedade atual, principalmente a agressão interpessoal. Entretanto esta modalidade de crime na sua maioria não resulte em morte, desencadeando um significativo número de sequelas, além de demandar maiores gastos públicos e privados (Barbosa et al., 2019). Deste modo, a violência estrutura uma série de consequências individuais e sociais, em especial aos jovens, caracterizados nas estatísticas como os que mais matam e os que mais morrem, é imperativo compreender a violência como um fenômeno multicausal, visto que ela está justaposta aos processos sociais, reforçados por uma rede de carências institucionais e estruturais, o que requer diagnósticos, planejamento, ações permanentes para seu enfrentamento e uma reflexão profícua sobre a perspectiva da complexidade dos contextos sociais (Pinto, Alpes \& Colares, 2019).

Dentro deste contexto, devido a sua posição de vulnerabilidade no corpo, a face, é uma região anatômica com foco de alta incidência em agressões físicas e acidentes. Segundo a Organização Mundial de Saúde (OMS) o trauma está entre as principais causas de morte e invalidez no mundo, afetando todos os povos com grande variabilidade epidemiológica. Tornando os ferimentos de face como um dos problemas mais significativos de saúde, devido ao seu percurso clínico de severa morbidade, perda de função, desfiguração estética, lenta reabilitação, abordagem multiprofissional e alto custo financeiro (Chaves et al., 2018; Torres et al., 2018). 
Em relação as agressões físicas, estas têm sido o principal fator etiológico dos traumas faciais, visto que a quantidade de lesões localizadas na face geralmente é maior, sendo ela o primeiro objeto de interação entre indivíduos, tornado uma região de baixa proteção e alta exposição (Hage et al., 2018). De acordo com a importância da prevalência dos traumatismos faciais de etiologia por violência interpessoal, o presente estudo tem por finalidade analisar os casos de traumatismos buco-maxilofaciais originados por agressões físicas.

Deste modo, os traumas maxilofaciais são frequentemente associados à morbidade em diferentes graus de dano físico, funcional e estético, representando um desafio para os serviços de saúde pública em todo o mundo, incluindo alto custo financeiro significativo para reparação de danos estéticos e limitações funcionais. Não obstante, a violência produz profundas consequências sobre a saúde, onde a maioria das vítimas são propensas a ter problemas psicológicos, síndromes de dor crônica, depressão e distúrbios psicossomáticos (Cabral, Lima \& Oliveira, 2021).

Contudo, os traumas buco-maxilo-faciais motivados por agressão física ocorrem mais no gênero masculino, predominando a segunda e terceira década de vida, majoritariamente no período da noite, soco ou chute são os principais mecanismos empregados, sendo uma pessoa desconhecida como o principal alvo envolvido, e a discussão, como o principal motivo desencadeador de tal violência (Ferreira et al., 2014).

Destarte, justifica-se este estudo por promover uma profícua atenção a estes dados que podem beneficiar a sociedade ao fundamentar estratégias governamentais preventivas para controlar os eventos que podem resultar em traumatismos desta etiologia.

\section{Metodologia}

O presente artigo trata-se de uma revisão integrativa, que é uma modalidade de estudo que proporciona uma síntese de conhecimento, direcionada ao cuidado clínico e ao ensino fundamentado na relevância das evidências científicas. Engloba desde a definição do problema clínico, a identificação das informações necessárias, a condução da busca de estudos na literatura, até a avaliação crítica e identificação da aplicabilidade dos dados coletados nas publicações selecionadas (Sousa et al., 2017).

Foi realizada uma pesquisa bibliográfica sobre o tema central que envolveu a seguinte pergunta norteadora: Qual o perfil epidemiológico do trauma buco-maxilo-facial das vítimas brasileiras de agressão física?

A partir da utilização das bases de dados PUBMED, LILACS, e SCIELO foi realizada a seleção de trabalhos entre setembro de 2020 a dezembro de 2020, utilizando os descritores padronizados pelo Descritores em Ciências da Saúde (Decs): Agressão; Lesões Maxilofaciais; Epidemiologia, e seus correspondentes em inglês. Em todas as combinações foi utilizado o operador booleano And. Todas as publicações foram incluídas na revisão integrativa, segundo as bases de dados supracitadas, sem recorte temporal até o ano de 2020. Por fim, nenhuma restrição de idioma foi aplicada, foram realizadas traduções para artigos diferentes do inglês.

Os estudos foram compilados inicialmente pelos títulos, refinados pelos resumos. Após leitura dos resumos, os artigos que indicavam corresponder ao objetivo desta revisão, foram lidos integralmente, e uma vez que preencheram os critérios de inclusão, participaram deste trabalho. Desta forma, utilizaram-se os seguintes critérios: estar em formato de artigo, possuírem título e resumo que se assemelhem aos objetivos do trabalho e estar publicado na íntegra. Excluíram-se todos os trabalhos que não corresponderam a estes critérios, conforme ilustrado na Figura 1. 
Figura 1: Etapas da coleta de dados de estudo.

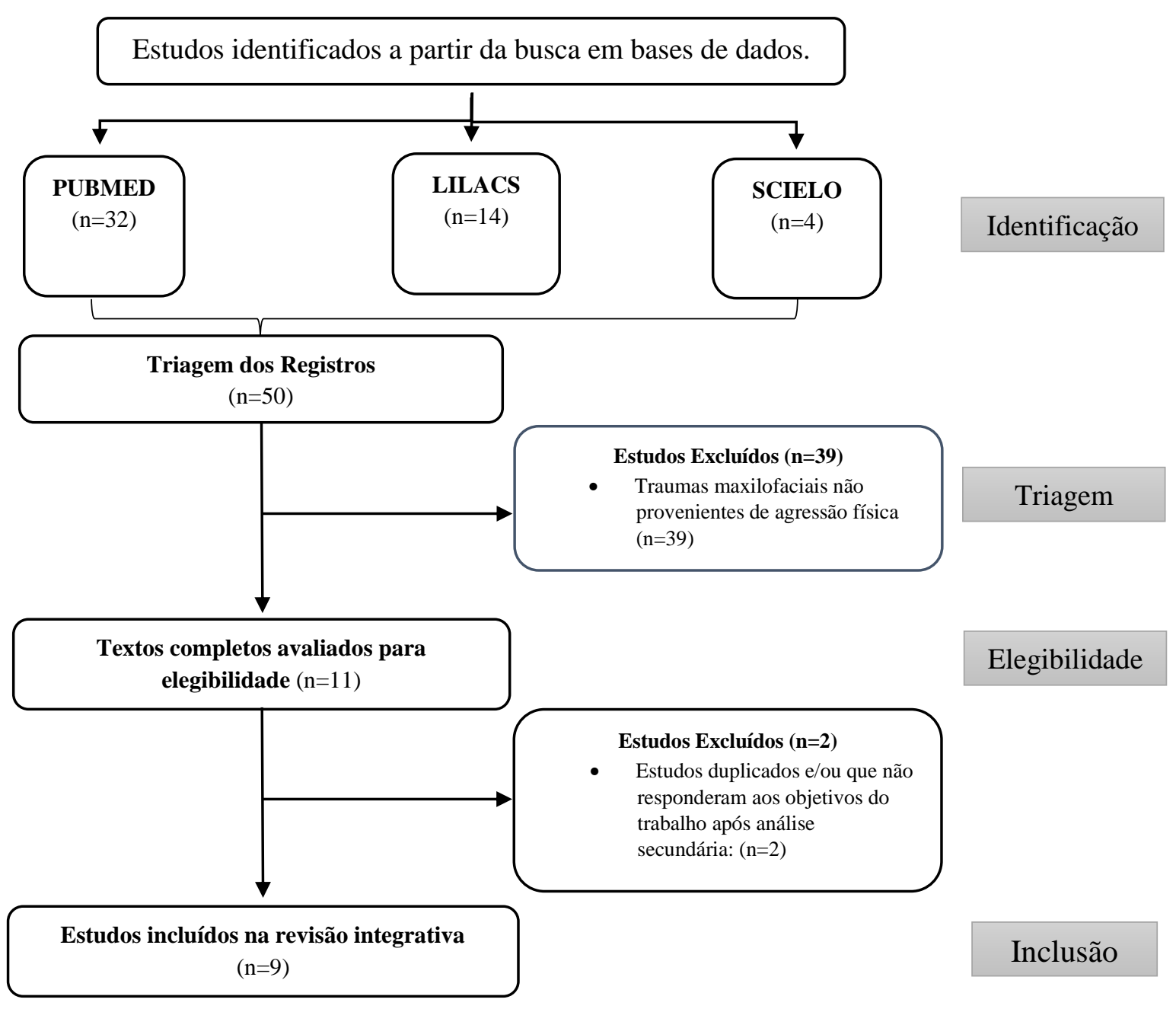

Fonte: Autores, (2021).

\section{Resultados}

Utilizando as palavras Agressão, Lesões Maxilofaciais e Epidemiologia, até o ano de 2020 foram encontrados um total de 50 artigos nas bases de dados PubMed, Lilacs e Scielo, sem restrição de idioma. Sendo 29 artigos excluídos na leitura de título, destes 16 da PubMed, 3 da Scielo e 10 da Lilacs. Dos 21 trabalhos que restaram, 10 foram excluídos na leitura de resumos. Ao final, 11 artigos estavam disponíveis para leitura na íntegra, onde destes, 2 foram descartados por duplicação. Ao final, restaram 9 publicações que estavam de acordo com os critérios de inclusão do presente estudo.

Os estudos analisados no Quadro 1, mostraram que os traumas intencionais, marcadamente relacionados as agressões interpessoais, são preocupações de grandes proporções na sociedade contemporânea, visto que os traumas buco-maxilo-faciais perfazem graves consequências biopsicossociais em suas vítimas, podendo ocasionar desde incapacidade temporária ou permanente a, até mesmo, o óbito. Afetando todos os povos sem distinguir sexo, idade, renda, nível de instrução ou região geográfica, embora com grupos de risco bem delimitados. 
Quadro 1: Síntese de publicações incluídas na revisão integrativa, segundo as bases de dados PubMed, Lilacs e Scielo, até o ano de 2020.

\begin{tabular}{|c|c|c|c|c|}
\hline TÍTULO E AUTORES & ANO & DELINEAMENTO & OBJETIVOS & DESFECHO \\
\hline $\begin{array}{l}\text { Maxillo facial injuries in } \\
\text { victims of violence at } \\
\text { school environment. } \\
\text { (Cavalcati) }\end{array}$ & 2009 & $\begin{array}{l}\text { Estudo } \\
\text { Observacional e } \\
\text { Retrospectivo }\end{array}$ & $\begin{array}{llr}\text { Investigar a presença de } \\
\text { lesões no complexo } \\
\text { maxilofacial em crianças e } \\
\text { adolescentes vítimas de } \\
\text { violência física } \\
\text { ambiente escolar. }\end{array}$ & $\begin{array}{l}\text { Apesar da pequena amostra analisada, } \\
\text { a existência de injúrias na cavidade } \\
\text { bucal em vítimas de agressão no } \\
\text { ambiente escolar mostrou-se elevada, } \\
\text { demonstrando ser importante a } \\
\text { realização de investigações futuras, } \\
\text { que busquem estudar a presença e a } \\
\text { distribuição de lesões no complexo } \\
\text { maxilofacial. }\end{array}$ \\
\hline $\begin{array}{l}\text { Levantamento } \\
\text { epidemiológico de cegueira } \\
\text { e perda do globo ocular por } \\
\text { agressões físicas e por } \\
\text { armas de fogo em uma } \\
\text { instituição pública de } \\
\text { ensino superior do Recife. } \\
\text { (Cardoso et al.) }\end{array}$ & 2010 & $\begin{array}{l}\text { Estudo } \\
\text { Retrospectivo } \\
\text { Quantitativo }\end{array}$ & $\begin{array}{l}\text { Determinar a prevalência } \\
\text { da perda do globo ocular } \\
\text { devido a agressões físicas e } \\
\text { armas a partir de } \\
\text { prontuários de pacientes } \\
\text { mutilados faciais atendidos } \\
\text { na Universidade Federal de } \\
\text { Pernambuco. }\end{array}$ & $\begin{array}{l}\text { Os casos de traumatismos por armas } \\
\text { de fogo foram mais prevalentes do } \\
\text { que por agressão física, tendo sido a } \\
\text { rua o principal espaço social para a } \\
\text { expressão da violência, destes o perfil } \\
\text { sociodemográfico e econômico } \\
\text { evidenciou que eram } \\
\text { predominantemente jovens e adultos } \\
\text { de até } 49 \text { anos e do sexo masculino. }\end{array}$ \\
\hline $\begin{array}{l}\text { What is expected from a } \\
\text { facial trauma caused by } \\
\text { violence? } \\
\text { (Goulart et al.) }\end{array}$ & 2014 & $\begin{array}{l}\text { Estudo } \\
\text { Retrospectivo }\end{array}$ & $\begin{array}{l}\text { Comparar as peculiaridades } \\
\text { das lesões maxilofaciais } \\
\text { causadas pela violência } \\
\text { interpessoal com outros } \\
\text { fatores etiológicos. }\end{array}$ & $\begin{array}{l}\text { O trauma facial por violência } \\
\text { interpessoal parece estar relacionado } \\
\text { a um maior índice de fraturas e } \\
\text { lacerações faciais quando comparado } \\
\text { a todos os pacientes com lesões } \\
\text { faciais. Áreas proeminentes da face e } \\
\text { neurocrânio foram mais afetadas. }\end{array}$ \\
\hline $\begin{array}{l}\text { Maxillofacial injuries as } \\
\text { markers of urban violence: } \\
\text { a comparative analysis } \\
\text { between the genders. } \\
\text { (Silva et al.) }\end{array}$ & 2014 & $\begin{array}{l}\text { Estudo Transversal } \\
\text { Exploratório }\end{array}$ & $\begin{array}{l}\text { Comparar a diferenças de } \\
\text { vitimização entre os sexos } \\
\text { a partir das lesões } \\
\text { maxilofaciais } \\
\text { marcadores de violência } \\
\text { urbana. }\end{array}$ & $\begin{array}{l}\text { Houve registro de } 7.063 \text { vítimas, das } \\
\text { quais 55,1\% envolveram violência } \\
\text { interpessoal. A maioria das vítimas } \\
\text { era do sexo masculino (71,2\%). } \\
\text { Fraturas múltiplas foram o tipo de } \\
\text { lesão que melhor caracterizou o perfil } \\
\text { de vitimização do sexo masculino em } \\
\text { relação às lesões de partes moles. }\end{array}$ \\
\hline $\begin{array}{l}\text { Assault-related } \\
\text { maxillofacial injuries: the } \\
\text { results from the European } \\
\text { Maxillofacial } r \text { Trauma } \\
\text { (EURMAT) multicenter } \\
\text { and prospective } \\
\text { collaboration } \\
\qquad \\
\text { (Boffano et al.) }\end{array}$ & 2015 & $\begin{array}{l}\text { Estudo Prospectivo } \\
\text { Multicêntrico }\end{array}$ & $\begin{array}{l}\text { Apresentar e discutir as } \\
\text { características } \\
\text { demográficas e os padrões } \\
\text { de fraturas maxilofaciais } \\
\text { relacionadas a agressões } \\
\text { relatadas. }\end{array}$ & $\begin{array}{l}\text { A fratura observada com maior } \\
\text { frequência envolveu a mandíbula, } \\
\text { seguida por fraturas do complexo } \\
\text { órbito-zigomático-maxilar e fraturas } \\
\text { orbitárias. Indicando o papel crucial } \\
\text { da ingestão do álcool nas fraturas } \\
\text { relacionadas por agressão física. }\end{array}$ \\
\hline $\begin{array}{l}\text { Interpersonal violence, } \\
\text { circumstances of } \\
\text { aggressions and patterns of } \\
\text { maxillofacial injuries in the } \\
\text { metropolitan area of } \\
\text { Campina Grande, State of } \\
\text { Paraíba, Brazil (2008-2011) } \\
\text { (Bernardino et al.) }\end{array}$ & 2017 & $\begin{array}{l}\text { Estudo Transversal } \\
\text { Exploratório }\end{array}$ & $\begin{array}{l}\text { Esclarecer as circunstâncias } \\
\text { das agressões e os padrões } \\
\text { de lesões maxilofaciais em } \\
\text { vítimas de violência } \\
\text { interpessoal. }\end{array}$ & $\begin{array}{l}\text { Foram identificados diferentes perfis } \\
\text { de vitimização. Onde o primeiro era } \\
\text { composto principalmente por homens } \\
\text { de diferentes faixas etárias, vítimas de } \\
\text { violência comunitária, o segundo } \\
\text { grupo era composto principalmente } \\
\text { por adolescentes (10-19 anos) de } \\
\text { ambos os sexos, vítimas de violência } \\
\text { interpessoal e sem padrão específico } \\
\text { de lesões e o terceiro grupo era } \\
\text { composto por mulheres adultas }(\geq 20 \\
\text { anos) vítimas de violência doméstica. }\end{array}$ \\
\hline $\begin{array}{l}\text { Pattern of oral-maxillofacial } \\
\text { trauma from violence } \\
\text { against women and its }\end{array}$ & 2017 & $\begin{array}{l}\text { Estudo Transversal } \\
\text { Exploratório }\end{array}$ & $\begin{array}{lll}\text { Caracterizar } & \text { o perfil de } \\
\text { mulheres } & \text { vítimas } & \text { de } \\
\text { violência } & \text { e } & \text { identificar }\end{array}$ & $\begin{array}{l}\text { A ocorrência de trauma maxilofacial } \\
\text { foi de } 46,4 \% \text {, a média de idade das } \\
\text { vítimas foi de } 29,38 \text {. Com base na }\end{array}$ \\
\hline
\end{tabular}




\begin{tabular}{|c|c|c|c|c|}
\hline $\begin{array}{l}\text { associated factors. } \\
\text { (Nóbrega et al.) }\end{array}$ & & & $\begin{array}{l}\text { fatores associados às lesões } \\
\text { maxilofaciais. }\end{array}$ & $\begin{array}{l}\text { árvore de decisão, o perfil da } \\
\text { violência contra a mulher pode ser } \\
\text { explicado pelo sexo do agressor e } \\
\text { características sociodemográficas das } \\
\text { vítimas, como estado civil, local de } \\
\text { residência e nível educacional. }\end{array}$ \\
\hline $\begin{array}{l}\text { Patterns of Oral- } \\
\text { Maxillofacial Trauma and } \\
\text { Determinant Factors among } \\
\text { Brazilian Victims of } \\
\text { Physical Aggression: A } 4- \\
\text { Year Study } \\
\text { (Barbosa et al.) }\end{array}$ & 2018 & $\begin{array}{l}\text { Estudo Transversal } \\
\text { Exploratório }\end{array}$ & $\begin{array}{l}\text { Determinar a prevalência } \\
\text { de trauma buco-maxilo- } \\
\text { facial entre vítimas } \\
\text { brasileiras de agressão } \\
\text { física e identificar seus } \\
\text { fatores associados a partir } \\
\text { de uma perspectiva } \\
\text { médico-legal e forense. }\end{array}$ & $\begin{array}{l}\text { A prevalência de trauma buco- } \\
\text { maxilofacial foi elevada e os fatores } \\
\text { associados foram área de residência, } \\
\text { tipo de violência, tipo de agressor, } \\
\text { mecanismo de violência, dia e } \\
\text { período de ocorrência. }\end{array}$ \\
\hline $\begin{array}{l}\text { Characterization of oral } \\
\text { maxillofacial } \\
\text { resulting from physical } \\
\text { aggression: differences } \\
\text { between genders } \\
\text { (Garcez et al.) }\end{array}$ & 2019 & $\begin{array}{l}\text { Estudo } \\
\text { Transversal } \\
\text { Exploratório }\end{array}$ & $\begin{array}{l}\text { Detalhar as lesões } \\
\text { maxilofaciais decorrentes } \\
\text { de agressão física em uma } \\
\text { capital do nordeste } \\
\text { brasileiro e analisar as } \\
\text { diferenças de gênero. }\end{array}$ & $\begin{array}{l}\text { Identificaram que a incidência de } \\
\text { lesões orais e maxilofaciais por } \\
\text { agressão física é elevada em São } \\
\text { Luís, no Maranhão, e embora as } \\
\text { mulheres sejam as mais acometidas, } \\
\text { as lesões maxilofaciais nos homens } \\
\text { são mais graves. }\end{array}$ \\
\hline
\end{tabular}

Fonte: Autores, (2021).

Ao visualizar os trabalhos realizados até o ano de 2020, deve-se considerar que as circunstâncias e as sequelas da violência urbana são bem documentadas, porém a identificação dos fatores etiológicos tem sido mais difícil, o que reflete a necessidade de conduzir estudos epidemiológicos de traumatologia maxilofacial mais robustos.

\section{Discussão}

Com o objetivo de explanar informações sobre perfil epidemiológico buco-maxilo-facial das vítimas de agressão física, buscou-se construir uma reflexão com os dados presentes na literatura científica, tanto sobre etiologia dessas lesões, como os impactos sociais desencadeados pela violência em nossa sociedade. É evidente que a assistência dos pacientes traumatizados não requer somente o conhecimento biomédico e intervenções tecnicistas, mas sobretudo uma compreensão ampla dos agentes indutores do trauma e consequentemente da violência interpessoal. Afinal, dentro do conceito multidisciplinar em saúde, tudo o que possa induzir uma ameaça ou agravo à vida, as relações interpessoais e a qualidade da existência versam sobre a saúde pública.

Segundo Cardoso et al. (2010); Silva et al. (2014); Nóbrega et al. (2017) e Garcez et al. (2019), as circunstâncias e resultantes da violência, principalmente localizada nos centros urbanos são vastamente relatadas, porém, os fatores etiológicos e padrões epidemiológicos das lesões que afetam o complexo maxilofacial precisam ser melhores descritas. Deste modo, Barbosa et al. (2018), asseveram que os traumas intencionais, relacionados principalmente as agressões físicas, são preocupações de grandes proporções contemporâneas, afinal as taxas de violência são crescentes em toda a sociedade e os traumas associados as agressões, vem atingindo proporções epidêmicas por todo o mundo.

Sabido que a região anatômica de cabeça e pescoço possui maior vulnerabilidade as exposições traumáticas devido a projeção anterior da face (Moura, Daltro \& Almeida, 2016). Goulart et al. (2014) e Boffano et al. (2015), destacam que as fraturas do complexo maxilofacial, isoladas ou correlacionadas a quadros de politraumatismo podem desencadear consideráveis danos morfofuncionais, impactando negativamente na qualidade de vida destes indivíduos, induzindo danos psicológicos, estéticos, econômicos e sociais, além de denotar uma rede ampla de assistência com altos custos no âmbito da saúde pública, que vão desde o tratamento inicial até a reabilitação. A divulgação destes dados reflete no reconhecimento das 
principais carências da vítima em termos de atendimento primário e de seguimento, possibilitando a determinação dos impactos dessas lesões, que em sua maioria perpassam as questões físicas.

Sobre a distribuição por sexo dos pacientes relatados nos estudos secionados, Silva et al. (2014), advogam que nos marcadores de violência urbana, tanto as mulheres como os homens, convivem com fatores de risco semelhantes. Embora, particularidades comportamentais podem ser utilizadas para justificar as peculiaridades de ambos, assim os indicadores de morbidade e mortalidade apontam situações de vitimização diferentes entre os sexos. Cavalcante (2009); Cardoso et al. (2010); Goulart et al. (2014); Silva et al. (2014); Boffano et al. (2015); Bernardino et al. (2017) e Barbosa et al. (2018), relatam que a maioria das vítimas eram do sexo masculino, de forma uníssona, afirmam a existência de maiores frequências de fraturas simples e múltiplas, quando confrontadas com os números de lesões em partes moles, evidencia que estes estão envolvidos em ocorrências mais graves e sobretudo, com potencial risco de morte entre pacientes do sexo masculino.

Em contrapartida, Nóbrega et al. (2017) e Garcez et al. (2019), relatam que em nosso país, a violência física é mais frequente perpetrada contra as mulheres, sendo apontada como a primeira ou a segunda principal causa de lesões maxilofaciais, neste grupo. Onde em seus estudos o sexo feminino compreendeu a maior parte das vítimas, contrapondo-se a maioria dos estudos analisados, que identificaram maior proporção de homens como vítimas de traumas maxilofaciais. A prevalência da violência doméstica, se sobrepôs a comunitária, onde majoritariamente os agressores eram do sexo masculino e com algum grau de vínculo pessoal ou emocional com as vítimas, o mecanismo de agressão mais comumente relatado foi a força física e grande parte dos casos ocorreu durante a noite. É imperativo destacar que a violência perpetrada nas mulheres é um problema social presente de forma latente em todas as categorias de idade, escolaridade, classe socioeconômica e religião, sendo responsáveis por imprimir graves cicatrizes físicas e emocionais. Embora, em ambos os estudos as mulheres foram mais acometidas por lesões no complexo bucomaxilofacial, o sexo masculino apresenta um padrão de lesões com aspectos de maior severidade.

Após análise cuidadosa dos estudos, Goulart et al. (2010); Silva et al. (2014); Nóbrega et al. (2017); Barbosa (2018) e Garcez et al. (2019), revelaram que a incidência das agressões prevaleceu entre a segunda e terceira década de vida, no período noturno, afinal é reconhecido pelo maior envolvimento social de indivíduos em bares, festas e outros eventos, facilitando, assim, o consumo de bebidas alcoólicas e maiores possibilidades de ocorrer violência interpessoal. Por conseguinte, é também no período da noite em que muitas mulheres são agredidas, ou a criminalidade é mais evidente.

Por sua vez, Cavalcanti (2009), relatou de maneira exclusiva a presença de lesões no complexo maxilofacial em crianças e adolescentes provenientes de violência física, inseridas no ambiente escolar. Conforme supracitado que a violência é um fenômeno observado em todos os domínios da vida social, e neles também incluídas escolas. Através de um estudo observacional e retrospectivo, o autor relatou quanto ao gênero que $61,9 \%$ eram do sexo masculino e $38,1 \%$ feminino, quanto à faixa etária mais de $80 \%$ possuíam entre treze e dezessete anos. Revelaram também que o agente agressor em 92,9\% dos perpetradores foram colegas da vítima. No que se refere à região do corpo atingida, as lesões na região da cabeça e face exclusivamente estiveram presentes em 50,0\% dos laudos, entretanto quando se verifica a existência de lesões nessa região associadas à outra parte do corpo o índice foi de $69,1 \%$, com $23,8 \%$ das vítimas apresentando injúrias na região oral, onde a totalidade destas lesões estiveram em tecido mole, principalmente nos lábios.

Portanto, as injúrias compreendidas no sistema estomatognático carecem ser avaliadas pelo profissional da Odontologia, com expertise legal para conduzir as perícias e atendimentos. Apesar da exclusividade do estudo e da pequena amostra, ficou evidente a presença elevada de injúrias na cavidade oral das vítimas de agressão inseridas no ambiente escolar, demarcando a necessidade de conduzir novas investigações, que busquem estudar de maneira mais robusta a distribuição de lesões no complexo maxilofacial (Cavalcanti, 2009). 
É sabido que as fraturas faciais podem variar de acordo com a região geográfica, densidade, período de estudo, influências socioeconômicas e características governamentais da população. Boffano et al. (2015) e Bernardino et al. (2017), asseveram a importância da comparação de estudos epidemiológicos com o intuito de esclarecer as principais semelhanças e divergências entre os resultados obtidos na distribuição das lesões de tecidos moles e duros de acordo com a região facial afetada. Estudos abrangentes sobre a epidemiologia do trauma facial como os de Wulkna, Parreira e Botter (2005); Cabral, Lima e Oliveira (2021) e Andrade et al. (2021) reforçam que as lesões na face decorrentes de agressão física se apresentam em casos de fraturas Le Fort, panfaciais ou complexas e sobretudo com fraturas de ossos isolados. Estes dados são relevantes, pois revelam que os traumas deformantes decorrentes de situações muitas vezes evitáveis.

Diversos estudos apontaram que os pacientes vítimas de agressão física cursaram com lesões de tecido mole na face, sendo a prevalência de traumatismos dento-alveolares como a mais controversa e abundante na literatura (Goulart et al. 2014; Barbosa et al. 2018). Sobre as fraturas ósseas recorrentes em agressões físicas, Garcez et al. 2019, relatam que o destaque é para o complexo anatômico nasal, região mandibular e do complexo zigomático-maxilar, marcadamente no terço médio da face.

As lesões no terço médio foram as mais frequentes em todos os estudos analisados, onde as regiões mais acometidas foram a orbitária, frontal e labial. Diferenças entre gêneros foram verificadas nas lesões labial, bucinadora e massetérica (Silva et al. 2014; Boffano et al. 2015 \& Bernardino et al. 2017). O osso nasal é o osso mais proeminente da face, sendo, portanto, um alvo facilmente atingido durante as agressões físicas, além de requerer uma força menor para fraturá-lo do que qualquer outro osso da face (Barbosa et al. 2018). Contudo, a sinergia com traumatismos corporais depende do tipo de mecanismo etiológico, em razão da quantidade de energia de impacto derivada em cada variedade de acidente. Por fim, Nóbrega et al. 2017, advogam que os instrumentos contundentes foram os mais observados, especialmente em mulheres, com lesões provocadas por instrumentos cortantes e perfuro contundentes, enquanto Bernardino et al. 2017, alegam que a combinação de chutes e socos como formas mais corriqueiras de agressões à face.

Sobre o mecanismo de agressão, Cardoso et al. 2010, desenvolveram um estudo retrospectivo e quantitativo, específico para determinar a prevalência da perda do globo ocular devido a agressões físicas e por armas de fogo. Considerando os indicadores sociodemográficos dos 53 pesquisados, a idade deles variou entre 11 e 84 anos, apresentando média de 41,26 anos, a amostra se constituiu majoritariamente de indivíduos do sexo masculino em 79,2\%. Dentre os avaliados, a enucleação foi a cirurgia mutiladora de maior incidência com 53,1\%. Sobre à etiologia, as perdas oculares foram causadas em 73,6\% dos casos por traumatismos induzidos por armas de fogo, enquanto $26,4 \%$ dos casos por agressão física, sido a rua o principal espaço social em 88,4\% para a expressão da violência. Destarte, a associação dos tipos de violência com cada uma das variáveis médico-odontológicas, indicaram que a queixa principal dos pacientes com perda ocular agredidos fisicamente era funcional em $38,5 \%$, já os vitimados por arma de fogo era devido à estética em $61,1 \%$ dos casos atendidos.

Por fim, os traumas maxilofaciais são frequentemente associados à morbidade em diferentes graus de dano físico, funcional e estético, representando um desafio para os serviços de saúde pública em todo o mundo, incluindo alto custo financeiro significativo para reparação de danos estéticos (Minari et al. 2020; Maia et al. 2021). Não obstante, a violência tem ainda profundas consequências sobre a saúde, além das limitações funcionais, as vítimas são propensas a ter problemas psicológicos, síndromes de dor crônica, depressão e distúrbios psicossomáticos (Silva, Gonzaga \& Barbosa, 2021). Entretanto a temática não tenha sido objeto principal de pesquisa nesta revisão, é unânime entre os autores confrontados a influência do consumo de álcool e outras drogas entorpecentes no desempenho de fatores importantes no incremento da violência, e por conseguinte, dos casos de agressão (Queiroz et al. 2021). 


\section{Considerações Finais}

Por fim, os traumas buco-maxilo-faciais por agressão física ocorreram mais no gênero masculino, predominando na faixa etária de 20 a 30 anos, majoritariamente no período da noite, soco ou chute foram os principais mecanismos das agressões, sendo que a pessoa desconhecida como o principal alvo envolvido, e a discussão, como o principal motivo desencadeador de tal violência. Desta forma, fica evidente a necessidade de uma profícua atenção a estes dados que podem beneficiar a sociedade ao fundamentar estratégias governamentais preventivas para controlar os eventos que podem resultar em traumatismos desta etiologia.

Destarte, compreendendo que as ações contra a violência devem ser assumidas por toda a sociedade, sugere-se que os profissionais em formação sejam mais bem preparados para detectar indícios de violência física em seus pacientes e encorajar a denúncia, afinal o mundo está sangrando e uma das formas de tentar conter esta hemorragia é cuidar das vítimas da violência urbana. Espera-se que os profissionais responsáveis pela elaboração dos laudos periciais padronizem a metodologia de coleta de informações, objetivando o registro do maior número de detalhes da agressão.

\section{Referências}

Andrade, M. J. H., Limoeiro, A. G. S., Souza, C. C., Nascimento, W. M., \& Moreira, D. C. (2021). Estudo Epidemiológico de fraturas faciais em uma subpopulação brasileira. Research, Society and Development, 10(5), e27910514937.

Barbosa, K. G. N., Walker, B. B., Schuurman, N., Cavalvanti, S. D. L. B., Ferreira, E. F., \& Ferreira, R. C. (2019). Epidemiological and spatial characteristics of interpersonal physical violence in a Brazilian city: A comparative study of violent injury hotspots in familial versus non-familial settings, 2012-2014. Plos One, 14(1), e0208304.

Barbosa, K. G. N., Bernardino, I. M., Cavalcante, G. M. S., Ferreira, E. F., Martins, R. C., \& D’Ávila, S. (2018). Patterns of Oral-Maxillofacial Trauma and Determinant Factors among Brazilian Victims of Physical Aggression: A 4-Year Study. Oral Health Prev Dent, 16(6), $483-489$.

Bernardino, I. M., Barbosa, K. G. N., Nóbrega, L. M., Cavalcante, G. M. S., Ferreira, E. F., \& D’Ávila, S. (2017). Violência interpessoal, circunstâncias das agressões e padrões dos traumas maxilofaciais na região metropolitana de Campina Grande, Paraíba, Brasil (2008-2011). Ciência \& Saúde Coletiva, 22(9), 3033-3044.

Boffano, P., Roccia, F., Zavattero, E., Dediol, E., Ugle, V., Kova, Z., Vesnaver, A., Konstantinovic, V. S., Petrovic, M., Stephens, J., Kanzaria, A., Bhatti, N., Holmes, S., Pechalova, P. F., Bakardjiev, A. G., Malanchuk, V. A., Kopchak, A. V., Galteland, P., Mjoen, E., Skjelbred, P., Bertin, H., Marion, F., Guiol, J., Corre, P., Loes, S., Lekven, N., Laverick, S., Gordon, P., Tamme, T., Akermann, S., Karagozoglu, H., Kommers, S. C., \& Forouzanfar, T. (2015). Assaultrelated maxillofacial injuries: the results from the European Maxillofacial Trauma (EURMAT) multicenter and prospective collaboration. Oral and Maxillofacial Surgery, 119(4), 385-391.

Cabral, C. L., Lima, M. O., \& Oliveira, S. M. L. (2021) Traumatismos faciais ocasionados por agressão física: uma revisão bibliográfica. Research, Society and Development, 10(1), e14110111616.

Cavalcanti, A. L. (2009). Lesões no complexo maxilofacial em vítimas de violência no ambiente escolar. Ciência \& Saúde Coletiva, $14(5), 1835-1842$.

Cardoso, S. O., Magalhães, T. S., Massa, M. E., Souza, J. C. A., \& Cardoso, J. O. (2010). Levantamento epidemiológico de cegueira e perda do globo ocular por agressões físicas e por armas de fogo em uma instituição pública de ensino superior do Recife. Revista de Odontologia da Universidade Cidade de São Paulo, 22(2), 111-121.

Chaves, A. S., Lund, R. G., Martos, J., Salas, M. M. S., \& Soares, M. R. P. S. (2018). Prevalência de traumatismos maxilofaciais causados por agressão ou violência física em mulheres adultas e os fatores associados: uma revisão de literatura. $R F O, 23(1), 60-67$.

Ferreira, M. C., Batista, A. M., Ferreira, F. O., Jorge, M. L. R., \& Marques, L. S. (2014). Pattern of oral-maxillofacial trauma stemming from interpersonal physical violence and determinant factors. Dent Traumatol, 30(1), 15-21.

Garcez, R. H. M., Thomaz, E. B. A. F., Marques, R. C., Azevedo, J. A. P., \& Lopes, F. F. (2019). Caracterização de lesões bucomaxilofaciais decorrentes de agressão física: diferenças entre gênero. Ciência \& Saúde Coletiva, 24(3), 1143-1152.

Goulart, D. R., Colombo, L., Moraes, M., \& Aspirino, L. (2014). What is expected from a facial trauma caused by violence?. Journal Of Oral \& Maxillofacial Research, 5(5), 1-7.

Hage, C. A., Xavier, T. B., Arantes, D. C., Zampiere, M. S., Nascimento, L. S. (2018). Traumas faciais e morbidade bucal provocada pela violência em Belém, estado do Pará, Brasil. Rev Pan-Amaz Saude, 9(1), 41-49.

Maia, A. B. P., Assis, S. G., Ribeiro, F. M. L., \& Pinto, L. W. (2021). The marks of gunshot wounds to the face. Braz J Otorhinolaryngol., 87(2), 145-151.

Moura, M. T. F. L., Daltro, R. M., \& Almeida, T. F. (2016). Traumas faciais: uma revisão sistemática da literatura. RFO UPF. $21(3)$, $331-337$. 
Research, Society and Development, v. 10, n. 12, e168101220127, 2021

(CC BY 4.0) | ISSN 2525-3409 | DOI: http://dx.doi.org/10.33448/rsd-v10i12.20127

Minari, I. S., Figueiredo, C. M. B. F., Oliveira, J. C. S., Brandini, D. A., \& Bassi, A. P. F. (2020). Incidência de múltiplas fraturas faciais: estudo retrospectivo de 20 anos. Research, Society and Development, 9(8), e327985347.

Nóbrega, L. M., Bernardino, I. M., Barbosa, K. G. N., Silva, J. A. L. S., Massoni, A. C. L. T., \& D’Ávila, S. (2017). Pattern of oral-maxillofacial trauma from violence against women and its associated factors. Dent Traumatol, 33(3), 181-188.

Pinto, M. P. P., Alpes, M. F., \& Colares, M. F. A. (2019). Situações de Violência Interpessoal/Bullying na Universidade: Recortes do Cotidiano Acadêmico de Estudantes da Área da Saúde. Revista Brasileira De Educação Médica, 43(1), 547-556.

Queiroz, D. R., Barros, M. V. G., Aguiar, J. A., Soares, F. C., Tassitano, R. M., Bezerra, J., \& Silva, L. M. P. (2021). Consumo de álcool e drogas ilícitas e envolvimento de adolescentes em violência física em Pernambuco, Brasil. Cadernos de Saúde Pública, 37(4), 1-10.

Silva, C. J. P., Ferreira, R. C., Paula, L. P. P., Haddad, J. P. A., Moura, A. C. M., Naves, M. D., \& Ferreira, E. F. (2014). Traumatismos maxilofaciais como marcadores de violência urbana: uma análise comparativa entre gêneros. Ciência \& Saúde Coletiva, 19(1), $127-136$.

Silva, M. K. A., Gonzaga, G. L. P., \& Barbosa, K. G. N. (2021). Traumatismos maxilofaciais em mulheres vítimas de violência física: Revisão de literatura. Research, Society and Development, 10(9), e55910918485.

Sousa, L. M. M., Vieira, C. M. A. M, Severino, S. S. P., \& Antunes, A. V. (2017). A metodologia de revisão integrativa da literatura em enfermagem. Revista Investigação em Enfermagem, 2(21), 17-26.

Torres, G. J. M., Tancred, T., Fletcher, A., Campbell, R., Thomas, J., \& Bonell, C. (2018). Integration of academic and health education for the prevention of physical aggression and violence in young people: systematic review, narrative synthesis and intervention components analysis. BMJ Open, 8(9), e020793.

Wulkan, M., Parreira, J. G., \& Botter, D. A. (2021). Epidemiologia do trauma facial. Revista da Associação Médica Brasileira. $51(5)$, p. 290-295. 